\title{
Case-Based Reasoning for Assessment and Diagnosis of Depression in Palliative Care
}

\author{
Agnar Aamodt ${ }^{1}$, Odd Erik Gundersen ${ }^{1,2}$, Jon Håvard Loge $\mathrm{e}^{3,4}$, \\ Elisabet Wasteson ${ }^{5,6}$, Tomasz Szczepanski ${ }^{2}$ \\ ${ }^{I}$ Dept. of Computer and Information Science, Norwegian University of Science and Technology \\ ${ }^{2}$ Verdande Technology \\ ${ }^{3}$ Dept. of Behavioral Sciences in Medicine, University of Oslo \\ ${ }^{4}$ National Resource Center for Long-term Effects after Cancer, Oslo University Hospital \\ ${ }^{5}$ Dept. of Cancer Research and Molecular Medicine, Faculty of Medicine, \\ Norwegian University of Science and Technology \\ ${ }^{6}$ Division of Psychology, Dept. of Social Sciences, Mid Sweden University, Campus Östersund \\ - on behalf of the European Palliative Care Research Collaborative (EPCRC)
}

\begin{abstract}
The goal of the research presented is to create a computational framework and system architecture for clinical decision support in palliative care. The application focused is the classification of depression. The method under investigation is case-based reasoning, motivated by the complexity of the domain and a lack of generalized principles of sufficient coverage and strength for diagnosis and treatment. A system architecture is described and exemplified through an implemented prototype. The outcome of the research so far is a system that captures the properties intended, and for which a clinical test set-up has been defined.
\end{abstract}

\section{Introduction}

A major focus in palliative care is to assess and treat the multiple expressions of the core disease that impair the patients' quality of life. The European Palliative Care Research Collaborative (EPCRC) [1] is a large EU-funded project in which an aim is to develop novel methods for assessment and classification of pain, fatigue (cachexia), and depression, for cancer patients in the palliative phase. The project has 11 partners, including many hospitals and academic medical centers across Europe. One line of research is the study of novel methods for utilization of computers in clinical practice. The work reported here is targeted at computerized decision support for identification of patients with depression - or more precisely: Major Depressive Episode (MDE).

Depression has been studied more than any other mental disorders in palliative care. Despite this fact, there are no agreed-upon methods on how to assess depression for clinical purposes. For classification of depression, the diagnostic systems ICD-10 or DSM-IV, which conceptually are quite similar, represent the "golden standards". However, as demonstrated in a recent review by The EPCRC consortium [2], most studies of depression in palliative care until now have not classified depression according to these systems. The resulting diversity in assessment and classification of depression in palliative care is reflected in the widely varying prevalence estimates (3\%-58\%) [23]. The inconsistency is confusing for the clinicians and can lead to over- and/or under-treatment, none of which are unproblematic for palliative patients with multiple symptoms and short life expectancies.

Evidence-based medicine is a well-established basis for medical practice. This is manifested by the increased emphasis of clinical guidelines and the development of computerized systems that support their utilization [3]. In order to enable reasoning and pro-active support in clinical decision support systems, the nature of medical and clinical knowledge needs to be taken into account. The medial domain is open, in the sense that 'established' knowledge and best practice may change, the cause-effect relations are often uncertain, etc. A large body of research has shown that it is extremely difficult to build a strong computational model based only on generalized knowledge for domains such as medicine (e.g. $[4,5])$. Another important source of information that clinicians make use of in their daily practice is the set of personal specific experiences gained through daily work. Past patient cases provide a level of specificity that focuses on single patients rather than generalized principles. Generalized and situation-specific knowledge therefore has a strong potential for effectively complementing each other. While our group is also studying such integrated approaches, the focus of the work reported in this paper is solely on the capture and utilization of the clinician's situation-specific experiences. 
The artificial intelligence field is studying how to utilize methods derived from cognitive science, including studies of human cognition. A method that has come out of this research is case-based reasoning (CBR) [6]. CBR supports a patient-centered approach to assessment, diagnosis, and treatment.

The paper first presents background information on the problem of diagnosing depression in palliative patients (ch 2), and on case-based reasoning for medical decision support (ch. 3). In ch. 4 we review some related research, which is followed by a presentation of our experimental system, in terms of its architecture and functional modules. In ch. 6 we illustrate the system's use, and in ch. 7 its test setup. In the final chapter the results are concluded.

\section{Assessment and classification of Depression}

The DSM-IV criteria for a Major Depressive Episode include the presence of at least five out of nine criteria: depressed mood, anhedonia, significant changes in weight or appetite, insomnia or hypersomnia, psychomotor agitation or retardation, fatigue or loss of energy, feelings of worthlessness or guilt, diminished ability to think, concentrate or indecisiveness, and recurrent thoughts of death. The criteria must be present during the last two week period, and also represent a change from previous functioning. At least one of the criteria present should be depressed mood or loss of interest or pleasure (i.e. anhedonia). Some of the criteria clearly overlap with symptoms of the cancer disease such as weight change or fatigue. Making a diagnosis of MDE in palliative care is on this background commonly perceived as a complicated task for the clinician.

In addition to the challenge related to the validity of some of the DSM-IV criteria, in particular significant changes in weight or appetite, fatigue or loss of energy and insomnia or hypersomnia, the optimal way to assess depression in the palliative care setting is as yet not established empirically. The best practice is therefore still the clinical interview in which the clinician decides to which degree the report from the patient fits with the over-all concept of MDE as defined by the DSM-IV/ the ICD-10.

Given the fact that the diagnostic criteria for MDE are established, the present uncertainty in palliative care as in relation to other physical diseases relates to the validity of each of the criteria and how to combine them into a valid diagnosis that can form the basis for therapeutic decisions in the palliative setting. There exists empirical data as to the optimal way to screen for depression. However, most clinicians perceive the existing methods for assessment of depression as complicated. This is probably a major contributing factor to the present under-detection and undertreatment of depression in the palliative setting.

At present the EPCRC activities on depression is running studies on the validity of the criteria for a major depressive order and optimal screening items(s) in the palliative setting. Hopefully these ongoing studies will provide us with better empirical data on the optimal definition of depression in the palliative care setting (i.e. the validity of each of the nine criteria). Improving the assessment methods is another part of the EPCRC project and the collaborative is working towards common methods for assessment of depression, pain and cachexia by use of computers.

The work reported here is based on the hypothesis that case-based reasoning can be a useful tool for decision support in palliative care, given the complexity that characterizes the palliative phase in terms of multiple and partly overlapping symptoms, a fluctuating course, and lack of consistent empirical data. Our ongoing work to improve the assessment and classification of depression is based on four steps:

(1) Literature study: A systematic literature review was conducted in order to identify which assessment methods and classification systems that have been used in studies of depression in palliative care [2].

The results show that although heterogeneity in assessments was expected, the diversity in the reviewed papers was more pronounced than anticipated. It was concluded that a variety of assessment methods had been used, and few studies classified depression by referring to a specific diagnostic system (such as DSM-IV or ICD-10) or by using cut-off scores. None of the included studies had used computers for the assessments.

(2) Experts' opinions: Seven experts on depression in cancer and palliative care were asked to evaluate the diagnostic criteria for their diagnostic relevance in palliative care, by use of the Delphi method. Results indicated that depressed mood, anhedonia, feelings of worthlessness and recurrent thoughts of death were considered the most relevant criteria. Supplementary symptoms such as hopelessness were appraised as important. In contrast, significant changes in weight or appetite and fatigue were evaluated as less relevant. Other symptoms were proposed by individual experts and examined by the panel.

(3) Patients' experiences: The contribution of the patients' subjective evaluation of depression has not been utilized in validation of the diagnostic criteria for depression in the palliative care setting. Thus, taking as starting point their experience with depression, previously depressed palliative cancer patients have been interviewed on their symptom experience as 
being clinically depressed. Preliminary results show that the patient's own experiences partly are congruent with the experts' opinions, but as would be expected the patients experiences were more heterogeneous. Additionally, the patients put a strong emphasis on two other aspects of depression namely anxiety symptoms (worrying and tension) and social withdrawal.

(4) Empirical data: As a joint data collection within the EPCRC, a computerized symptom assessment study (CSA) is collecting data on pain, cachexia and depression in more than 1000 European palliative patients. The overall objective for the work on depression is to examine the validity of the diagnostic criteria for depression measured by the self-report assessment-tool PHQ PRIME-MD. This instrument includes the DSM-IV criteria for MDE and also items on duration and functional consequences required for establishing a diagnosis of depression according to the DSM-IV. The instrument has diagnostic validity comparable to a clinician-administered interview [7]. The criteria's relationship to pain, physical function, performance status and other somatic manifestations of the cancer disease are analyzed in order to select the optimal items on depression and other aspects of the patients' condition to be included in the computerbased assessment-tool. A case-base generated from the CSA-study data is utilized in the pilot-version of the computerized tool for assessment of depression.

\section{Clinical decision support through case- based reasoning}

In CBR a computer model is built up of a set of concrete past situations, called cases, stored in a knowledge base referred to as a case base. A case in its basic form has two parts, a problem description part and a problem solution part. The problem description part constitutes the set of input features to the reasoning process, while the problem solution part is the output from the system's reasoning process and hence the system's suggested solution to the problem. A third part is often added: Outcome, i.e. the result after having applied the solution to the problem.

Reasoning methods of similarity assessment, pattern recognition, and analogical mapping, rather than theory-driven methods, operate over this case base of cases. In CBR a new problem is solved, or a state is interpreted, by finding a past case which problem description matches the current state to a sufficient degree. The problem solution part of the past case is then reused, either by reapplying to the past solution as it is, or modifying it to better fit the current situation.

Although the study reported here is focused on the case-based reasoning method, ongoing work in our group, within the medical domain, also include combining CBR with probabilistic reasoning within a causal model [8], and meta-level reasoning over a set of different reasoning methods [9]. There is ample evidence that clinicians partly reason from theoretical knowledge, and partly from case-specific or prototypebased experience, to some extent depending on how strong causal theories the particular medical area is supported with [10]. This is also reported from studies on mental disorders with reference to use of DSM-IV $[11,12]$. Given that the area of depressive disorders does not possess a strong causal theory, clinical reasoning will to some degree be based on similarity assessment and pattern comparison with a set of features.

A condensed overview of some CBR applications in medicine is given by Holt et. al. [13]. In [14] a status overview is given of some systems and methods. Below, a few relevant systems that utilize CBR for medical decision support are summarized.

The Care Partner system [15] integrates automatic processing of medical practice guidelines with CBR. The system uses specific cases and (generalized) prototypical cases to represent the variety and complexity of knowledge in the domain of stem-cell transplantation. It was developed in cooperation with Fred Hutchinson Cancer Research Center in Seattle, USA.

In the system Auguste [16] the utility of CBR for support of medical treatment planning in a domain lacking a strong domain theory was demonstrated. The system's task was to assist in planning of the ongoing care of Alzheimer's Disease patients. One of the goals was to better understand the reasoning processes used by health care professionals in this task. The role of the CBR part was to suggest whether a neuroleptic drug should be administered. A case was described with a set of features manually extracted from medical charts.

ISOR [17] is a system for long-term therapy support in psychiatry. It has also been applied to endocrinology. A generic method for therapy inefficacy has been developed, and tried out on several applications, including Alzheimers, Hypotyroidea, Eating disorders, and also Depression. In tests the system has performed at the level of experienced clinicians.

GLARE [18] is a general decision-support system for managing and utilizing clinical guidelines, in which a CBR system is being incorporated to handle situations that are not covered by the guidelines, socalled non-compliances. The CBR system will provide decision support in situations not sufficiently covered by the guidelines, or situations where the clinician disagrees with the guidelines. 


\section{System overview}

The role of our prototype system is to cover all clinician's tasks from the first patient encounter through diagnosis, treatment, and follow-up. This has been realized through two modules (see Figure 1): Gathering patient data is done through a dialogue with the patient and interaction with a nurse through the Assessment module while the CBR Decision support module supports the clinician with diagnosis. The Assessment module is based on the Assessment Tool, which was developed earlier in the EPCRC project, and has been used over some time, and by many project partners, to collect data in the more general CSA data collection mentioned in ch. 2 .

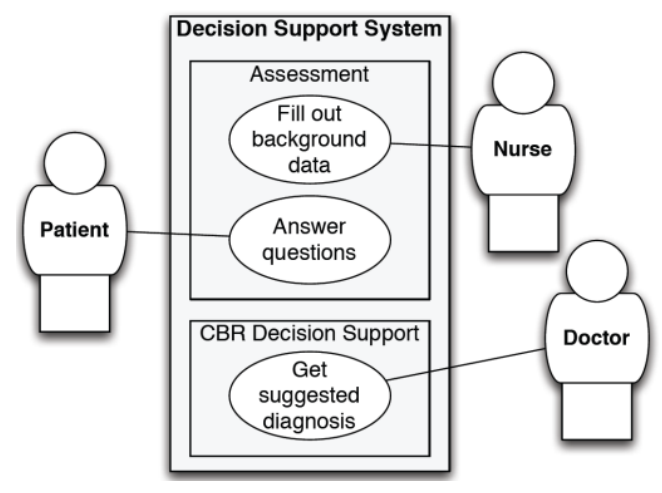

Figure 1. System modules and person roles

As Figure 1 illustrates, the patient fills in information in a predefined questionnaire, with some help of a nurse if needed, and additional patent administrative data are filled in by the nurse. The clinician will typically also add information to the patient description. The system provides the clinician with a suggested diagnosis and a possible treatment.

A case in the system represents a patient's session in a clinic. The information gathered from all three person roles make up the descriptive part of the new case. It includes static background data and patient journal data, in addition to answers to questionnaires: The PHQ PRIME-MD (as earlier described), and ESAS (Edmonton Symptom Assessment Scale), which assesses 9 commonly experienced symptoms of palliative care patients including anxiety and depressed mood. For the solved cases stored in the case base, the solution part of the case includes the diagnosis and treatment given to the patient represented by the past case. The system represents the DSM-IV criteria as a set of generalized patterns, which are made operational by being linked to a set of specific patient cases. The patient cases therefore contain more information than just answers to the nine DSM criteria, enabling a closer match to similar past situations.

A patient visit is handled by the system as follows: Patient data are entered forming the input case, which is matched with all the past cases in the case base. Similar cases are retrieved, and based on the best matched case a diagnosis is suggested. The proposed diagnosis is evaluated by the physician, and after a case has been found which diagnosis the clinician accepts, a treatment is suggested. The system learns by either storing a session just completed as a new case, or by updating case indexing knowledge. This process is illustrated in Figure 2, as an adapted CBR cycle [6]. Patient data builds up a new case, with an empty solution part, which gets filled by reusing information from the most similar past case.

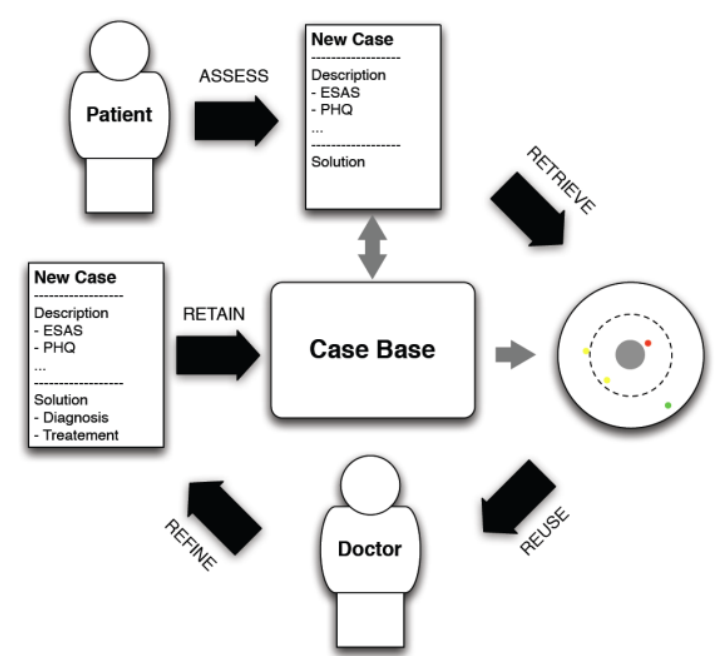

Figure 2. The CBR-supported decision cycle.

The results from the retrieval process is displayed on what we refer to as the "case radar" (the circle at middle right in Figure 2), which is a part of the underlying CBR software used for implementation. Our implementation has been made by using an existing commercial CBR system developed by Verdande Technology: the Edge platform. It is mainly being used within the application area of oil well drilling, where the specific tool is called DrillEdge [19]. For our purpose it has been adapted with some modifications to the medical domain.

\section{Diagnosis support - an illustration}

Focusing on the diagnostic support module, the data collected from the assessment modules enable the system to construct a new case. A graphical visualization of all cases that match the new case with a similarity above a given threshold are displayed on 
the case radar. This is illustrated in Figure 3. On the radar screen, the current situation, i.e. the new case, is in the centre, and a past case with a match above the threshold is shown as a colored dot on the radar. The distance from the centre is a measure of the similarity the most similar being nearest to the centre - and the color indicates the diagnosis of the patient represented by the past case.

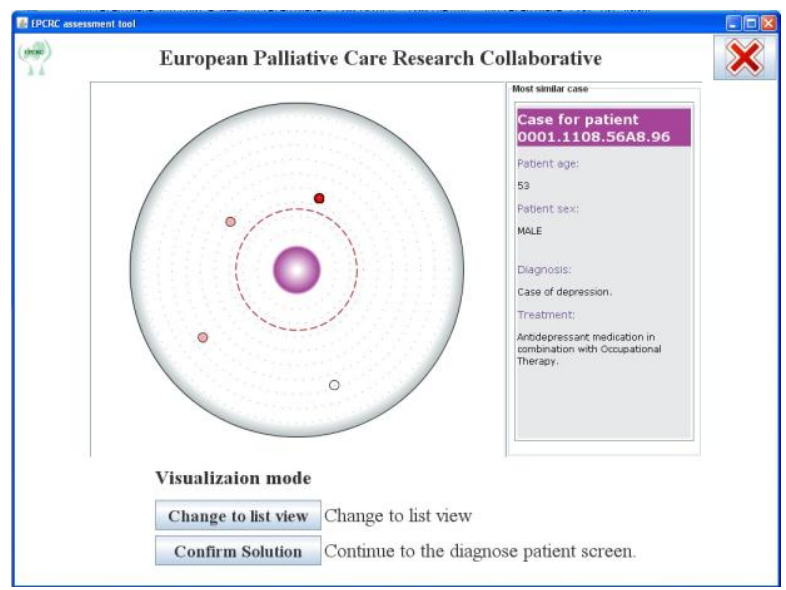

Figure 3. The case radar

Clicking on the case dot brings up a summary of the case (to the right in the Figure 3 screenshot). By clicking on the appropriate button (below the radar) the clinician has the option to inspect a case in more detail. The list view of a case is partially shown in Figure 4, in which details of the current (new) case are displayed. By clicking the "Case Match" tab, details of all the cases on the radar are displayed.

\section{Evaluation}

The quality of the diagnosis made by the system will be tested through a quantitative leave-one-out cross validation test, using the EPCRC CSA data, as well as through a comparison between the CBR system and the diagnosis made by clinicians on new patients. The cases in the CBR system contain a case description gathered from actual patient data collected as a part of the EPRC CSA study. These data do not contain a depression diagnosis, so a case solution (diagnosis and treatment) has been added by specialist clinicians from inspecting the data. The CBR system's performance will be compared to that of a clinician, tested on a set of new patients for which the clinician's diagnoses will be based on patient interviews.

The goal of the testing is to assess the usefulness for an active decision support system such as the one described, including the clinical effect of storing and reusing past patient episodes - i.e. the CBR method.
Whether our test will be able to say something about the adoption of computers for decision support in general, is less likely.

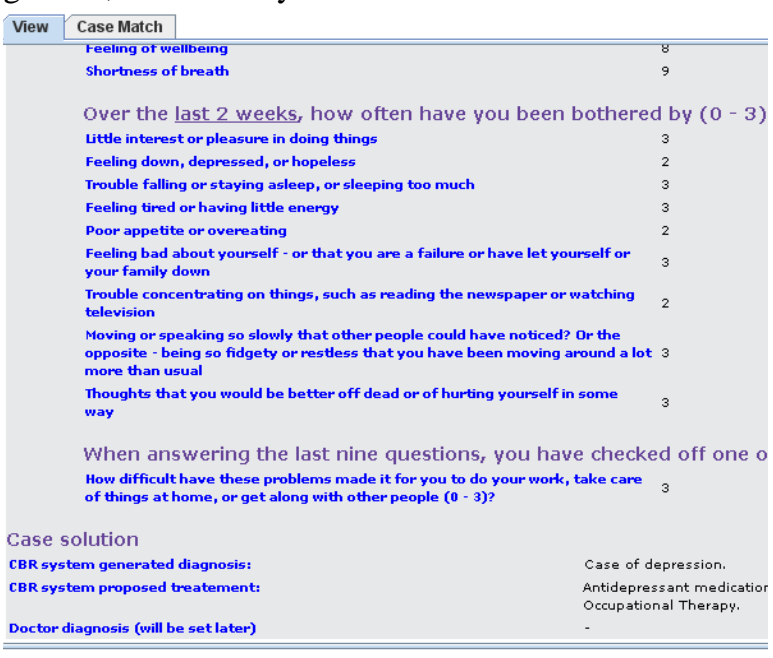

Figure 4. List view of extended case information.

Case-based reasoning, as a method applied to computerized support in medicine, is of course subject to the assessment and critique addressing computerization of clinical routines in general. Several studies have looked at the effect of introducing computerized tools in the clinic, compared to manual or paper-based tools. (e.g. [20, 21]). So far, no conclusive results have been obtained at the general level, although more specific effects have been observed related to particular types of operations that favor one or the other. A severe difficulty in these types of evaluations is to isolate the particular functionality of the system as such from the other effects of it being used [22]. A particular instantiation of a computer system, of which the human subjects are unfamiliar, and which may be more or less user-friendly and work-flow fitted, is often compared to a well known manual procedure. Hence, the generalization of the results from these types of studies, beyond the role of the specific system used in the study, is problematic and have to be made with great caution.

\section{Conclusion}

From an analysis of the problem of correctly diagnosing patients with Major Depressive Episode, a case-based approach to active decision support has been developed, and an experimental system implemented. The system has shown to possess the properties intended, including an informative graphical user interface. The next step is to evaluate the system in a clinical setting. The test setup has been defined, and data collection is now in process. 


\section{Acknowledgements}

The European Palliative Care Research Collaborative is funded by the European Commission's Sixth Framework Programme (contract no LSHC-CT2006-037777, EPCRC) with the overall aim to improve treatment of pain, depression and fatigue through translation research.

\section{References}

[1] Haugen, D.F., Kaasa, S.: The EPCRC. Eur J Palliat Care (2007; 14(3):130)

[2] Wasteson E, Brenne E, Higginson I.J., Hotopf M, LloydWilliams M, Kaasa S, Loge J.H.; Depression assessment and classification in palliative cancer patients: a systematic literature review. Palliat Med. Dec;23(8):739-53. 2009.

[3] Terenziani, P., Molino, G., Torchio, M.: A modular approach for representing and executing clinical guidelines. Artificial Intelligence in Medicine, 23(3):249\{276 (2001).

[4] Patel, V.L., Kaufman, D. R., Arocha J. F.: Emerging paradigms of cognition in medical decision-making. Journal of Biomedical Informatics, Volume 35 , Issue 1, Pages: 52 75,2002 .

[5] Schmidt, R, Motani, S., Bellazzi, R., Portinale, L. \& Gierl L., 2001. Case-Based Reasoning for Medical Knowledgebased Systems. International Journal of Medical Informatics 64 (2-3):355-367.

[6] Aamodt, A. and Plaza, E. Case-Based Reasoning: Foundational issues, current state, and future trends. AI Communications 1994, pp 1-21.

[7] Spitzer, R. L., Wakefield JC. (1999) DSM-IV diagnostic criterion for clinical significance: does it help solve the false positives problem? Am J Psychiatry. 1999 December; 156 (12): 1856-64.

[8] Bruland, T., Aamodt, A., and Langseth, H.: Architectures for Integrating Case-Based Reasoning and Bayesian Networks. To be published in Proceedings of The 6th International Conference on Intelligent Information Processing, Manchester UK, October 2010. Springer.

[9] Houeland, T.G. Aamodt, A.: Towards an Introspective Architecture for Meta-level Reasoning in Clinical Decision Support Systems. Proceedings of the Workshop on CBR in the Health Sciences, ICCBR 2009, Seattle, July 2009. pp. 235-244.

[10] Ahn, W., Kim, N.S.: Causal Status as a determinant of feature centrality. Cognitive Psychology, 41, 2000. Pages $361-416$.

[11] Kim, N.S., Ahn, W.: Clinical Psychologists' TheoryBased Representations of Mental Disorders Predict their
Diagnostic Reasoning and Memory. Journal of Experimental Psychology, 131, 2002. Pages 451-476.

[12] Westen, D., Shedler, J., Bradley, R.: A prototype approach to personality disorder diagnosis. American Journal of Psychiatry 163:5, May 2006. Pages 846-856.

[13] Holt, A., Bichindaritz, I., Schmidt, R., and Perner, P.: Medical applications in case-based reasoning, The Knowledge Engineering Review, Vol. 00:0, 1-4. c 2005, Cambridge University Press.

[14] Bichindaritz, Isabelle (Ed.). Artificial Intelligence in Medicine, Special Issue on Case-Based reasoning in the health sciences, Vol. 36, 2005.

[15] Bichindaritz, I., Kansu, E., Sullivan, K.M.: Case-Based Reasoning in CARE-PARTNER: Gathering Evidence for Evidence-Based Medical Practice. EWCBR'98, LNAI 1488, pp. 334-345 (1998).

[16] Marling, CR \& Whitehouse, PJ, 2001. Case-Based Reasoning in the Care of Alzheimer's Disease Patients", In the Proceedings of the International conference of case-base reasoning, ICCBR-01 Springer- LNAI Vol 2080:702-715.

[17] Schmidt, R., Vorobieva, O.: Case-based reasoning investigation of therapy inefficacy. In A. Macintosh, R.Ellis, T. Allen (eds.): Applications and Innovations in Intelligent Systems XIII. Springer, 2006. Pages 13-25.

[18] Montani, S.: Case-based reasoning for managing noncompliance with clinical guidelines. ICCBR 2007, 5th Workshop on CBR in the Health Sciences (2007), NIH, National Institute of Health, National Library of Medicine: Introduction to the UMLS

[19] Verdande Technology: Harvesting knowledge to improve drilling performance in real time. Downloadable from page: http://www.verdandetechnology.com/research-apublications/drilledge-whitepaper.html

[20] Trivedi, M., et. al.: Barriers to implementation of a computerized decision support system for depression: an observational report on lessons learned in "real world" clinical settings. BMC Medical Informatics and Decision Making, 9:6, 2009. Pages 1-9.

[21] Bergman, L.G., Fors, G.H.: Decision support in psychiatry - a comparison between the diagnostic outcomes using a computerized decision support system versus manual diagnosis. BMC Medical Informatics and Decision Making. 8:9, 2008.

[22] Moehr, J.R.: Evaluation; salvation or nemesis of medical informatics. Computers in Biology and Medicine, 32 (2002), 113-125.

[23] Stiefel, R., Die Trill M, Berney, A.: Depression in palliative care: a pragmatic report from the Expert Group of the European Association for Palliative Care. Support Care Cancer 2001; 9:477-488. 